\title{
The use of data protection regulatory actions as a data source for privacy economics
}

\author{
Aaron Ceross and Andrew Simpson \\ Department of Computer Science, University of Oxford \\ Oxford, United Kingdom \\ firstname.lastname@cs.ox.ac.uk
}

\begin{abstract}
It is well understood that security informatics is constrained by the availability of reliable data sources, which limits the development of robust methods for measuring the impact of data breaches. To date, empirical data breach analysis has largely relied upon the use of economic and financial data associated with an organisation as a measure of impact. To provide an alternative, complementary approach, we explore monetary fines resulting from data protection regulatory actions to understand how the data can inform the evaluation of data breaches. The results indicate where context matters and also provide information on the wider challenges faced by organisations managing personal data.
\end{abstract}

\section{Introduction}

By most accounts, instances of data breaches have continued to rise in the past decade, yet the scope and range of data concerning these events has remained difficult to obtain [6]. While it is understood that the causes for these data breaches are rooted in a culmination of failures in an organisation's security and privacy measures, ranging from technical implementation [11] to organisational approaches to risk [12], defining generalisable metrics has proved challenging [15]. To this end, the impacts of data breaches have been measured in economic terms, considering the expenditure and loss of earnings [10]. In order to establish costs for a data breach, past studies such as [2] and [14] have extrapolated the financial burdens that a data breach event entails, supplemented by press releases, public financial data and surveys. Such studies inevitably have limitations in the kinds of conclusions that may be drawn, as the sample sizes tend to be small, with few variables to consider.

We consider monetary penalties from a data protection authority as a potential, complementary data source through examining data breach costs related to the regulatory fine. The data protection authority in question (the United Kingdom's Information Commissioner's Office (ICO)) examines a wide range of cases, giving rise to a richer data set than might be available 
through, for example, a voluntary survey. This may offer several benefits. First, it provides an accessible alternative source of data to investigate consequences. Second, it has the potential to provide a wider perspective with regards to characteristics of data breaches. By using information related to monetary penalties for data breaches from the ICO, we seek to address three questions: what are the features of the cases in which the data protection authorities has levied fines?; which of these features are correlated with the quantum of the fine?; and what are the trends, if any, of regulatory action? The results of this work contribute to the literature relating to the empirical analysis of data breaches. More importantly, while this work is inevitably limited in scope, as it is restricted to one national data protection authority, we perceive the work as illustrating the potential of a novel data set - regulatory fines - in examining data breaches.

\section{Background}

\subsection{Regulatory action in data protection is a recognised cost}

Regulatory action regarding failures to adequately protect personal data have been increasing. Such actions are features across a number of jurisdictions, and it is becoming increasingly difficult to ignore them. To enact such penalties, the relevant authorities are empowered to investigate the nature and causes of the data breach. The imposition of a penalty, as well as the quantum of such a penalty, may be viewed as a metric when attempting to analyse the impact of a failure of data security. Such penalties and the consequent analysis of such does, of course, perform another role: to act as a driver toward the adoption of better organisational and technical practices for data security.

The European Union has adopted the General Data Protection Regulation (GDPR), which will replace Member State implementation of the Data Protection Directive. ${ }^{1}$ The GDPR has stringent penalties, including a maximum fine of $€ 20$ million or $4 \%$ of global turnover of the preceding financial year, whichever is greater. In the US, past fines have been substantial, with, for example, $\$ 800,000$ being levied for improper data collection, ${ }^{2}$ as well as a $\$ 1.6$ million fine for the loss of 36 million user details in a data breach. ${ }^{3}$

\footnotetext{
${ }^{1}$ The Data Protection Directive (Directive 95/46/EC) required EU Member States to harmonise national legislation on data protection.

${ }^{2}$ https://www.ftc.gov/news-events/press-releases/2013/02/ 


\subsection{The Information Commissioner's Office}

The ICO is the data protection authority for the UK, tasked with ensuring that data controllers collect, process and store personal information in a secure manner that protects the informational privacy rights set out in the Data Protection Act of 1998 (DPA, or 'the Act'), the UK's implementation of the EU's Data Protection Directive. ${ }^{4}$ Within the legislation, a data controller is any recognised entity that determines the purposes and manner in which personal data are processed. Within the DPA, the personal data of an individual includes any information that allows for identification of the individual. The Act also describes a special category of personal data called sensitive personal data, which relates to specific attributes including health information, sexual orientation, religious and political beliefs.

There is no general obligation for data controllers to report data breaches to the ICO. A consequence of this is that the ICO primarily gathers intelligence on contraventions to the DPA through an online selfreporting system. In most cases, the reporter is the data controller itself, who has the responsibility to ensure the security of the records. The DPA empowers the ICO to issue monetary penalties of up to $£ 500,000$ for violations of the duties and obligations described in the Act. In determining a quantum for a monetary penalty, the size, sector and resources of the data controller are taken into account [7]. The ICO does not give a penalty for every reported instance of data breach. To be liable for a fine, the data controller must have failed in observing the data security principle of the DPA, which pertains to the maintenance of security of personal data. If it has been determined that a cases exists, the investigators will further consider: (i) the type of data lost; (ii) the cause of the breach; and (iii) the number of individuals affected.

\subsection{Related work}

A survey of the existing literature on empirical analysis of data breaches highlights two consistent themes: a scarcity of data sources and the types of data contained in such sources; and that the lack of robust data sources limits the types of analysis that may be conducted, restricting the scope of available measures of impacts of data breaches.

Data tends to come from three main sources: news media, surveys, and open data sets derived from a combination of the previous two. The lack of relevant, robust data affects the types of analysis and the resultant

\footnotetext{
${ }^{4}$ The legislation may be found at http://www.legislation.gov.uk/ukpga/1998/29/ contents.
} 
conclusions, particularly when attempting to derive validated, generalisable metrics $[6,15]$. Empirical analysis of data breaches has therefore been limited by data sources in the sort of analysis that may be undertaken. Various studies make use of news media to identify the number and specifics of cases (see, for example, [1] and [3]). A limitation of this approach is that the scope of the data set is curtailed by the interest of the media, which may only report on larger entities or larger number of records lost, as these may be more 'newsworthy'. These may not represent all types of data processing entities, nor all types of losses. Often this type of study achieves a data set of tens of firms. In a similar fashion, the UK Government publishes an annual Cyber Security Breaches Survey report. In 2016 's study, ${ }^{5}$ the methodology included a random probability survey of 1,008 businesses within the country, followed up with qualitative interviews with 30 respondents. A limitation of this approach is that the information derived is fixed to the data controller's perspective, ignoring the fact that there may be other factors that businesses do not consider. Further, the qualitative analysis reflects only those firms who wish to disclose practices and experiences, which may result in a biased view of widespread practice.

Much of the work on data breach impact has focused on event studies, which measure how the market reacts to announcement of a security event. The intuition is that a larger firm with a larger breach will result in a more significant financial impact. Cavusoglu et al. [3] concluded that the size and type of firm experiencing a data breach has an effect on the market's valuation of the firm. However, it has been shown that it is not necessarily possible to use this to predict trends within data breaches. Edwards et al. [5] acknowledged that, while there exist a number of data breach reports purporting to track the trends in data breaches, the variance in the data used produces faulty results when aggregated. Furthermore, the magnitude of the effect has also been shown to be variable. Acquisti et al. [1] provided an early empirical study on data breaches, assessing whether it was feasible to ascribe a cost to a violations of privacy derived from data breaches. The results showed that, while a firm experiences a statistically significant negative impact from a data breach, the effect is moderate in respect to expectation. Campbell et al. [2] found that sensitivity of the information subject to a data breach was significant in assessing the economic impact on a firm. Relatedly, in this paper we do not evaluate stock prices or extrapolate wider costs, but instead attempt to identify the attributes of reported data breaches and use the fine received as a measure of impact.

\footnotetext{
${ }^{5}$ https://www.gov.uk/government/publications/

cyber-security-breaches-survey-2016
} 


\section{Methods and data description}

Our study makes use of two data sets provided by the ICO. The first data set is the civil penalties data set, which includes all issued fines for data breaches. This is regularly updated with the ICO's regulatory actions and is available on the ICO's website. ${ }^{6}$ The data set includes all civil penalties issued by the ICO from November 2010 to 4th March 2017. This includes a total of 118 entries within the data set. In addition to the civil penalties data set, there is also a data set on reported breaches to the ICO between April 2015 and March 2016. The data set is the result of a freedom of information request [8]. The data contains reported data breaches, the sector in which the data controller operates, and the type cause of the breach. The provided information covers data breaches in 21 sectors and 12 causes. It contains neither information regarding the type of personal data affected nor the number of records.

For the purposes of this study, we use a more data protection-focused definition of the term data breach - which we consider to be any access or use of data that is not intended by the data subjects or the data controller. This requires a loss of security or control over the data by an entity other than the data controller. Other misuses of data, for example unauthorised processing, are included within this definition, as it allows for the inclusion of improper disclosures, wherein a data controller inadvertently sends personal information to other entities. We use exploratory data analysis to examine the civil penalties and data incident reporting data sets, with a view to examining the relationships between the categorical variables relating to sector, cause and category of personal data, as well as between the number of records lost and the fines received. The data analysis gives summary statistics with regards to the quantum of fines levied, as well as to the records that were affected by the breaches. We are also interested in the density of the fines. There are also frequency tables for the sectors in which the data controllers operate, the ascribed causes of the data breach, and the category of breached data.

\section{Results}

\subsection{Monetary penalties}

As of March 2017, the ICO has levied $£ 6.8$ million in fines for data breaches, from a total of 60 instances. As described in Section 2.2, the

\footnotetext{
${ }^{6}$ https://ico.org.uk/media/action-weve-taken/csvs/1042752/

civil-monetary-penalties.csv
} 

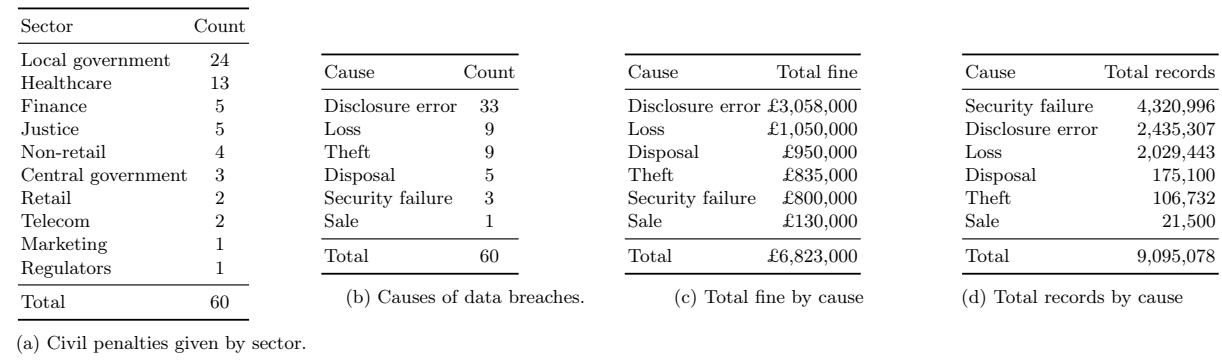

Table 1: Description of sectors (a), causes (b), fines (c), and records (d).

value of each fine reflects the severity of the breach, taking into account the resources of the data controller, and serves as a deterrent against poor data management practices. The fines range from $£ 5,000$ to $£ 400,000$, with a mean fine of $£ 113,717$. The ICO has not yet used its maximum possible fine of $£ 500,000$. Examining the count of causes in Table 1b shows that, out of issued fines, disclosure error accounts for more than half of the data breach causes (55\%), having more instances than all other categories combined. The next top three causes concern management of hardware and physical media (loss, theft, and improper disposal) - constituting $38 \%$ of fines. Security failures account for $5 \%$ of cases, having been the cause of only three instances.

The civil penalties data set captures information pertaining to data breach incidents from nine different sectors (Table 1b). Almost two-thirds of the data breach incidents $(61 \%)$ come from two sectors: local government and healthcare. The local government authorities account for the largest share $(40 \%)$, followed by healthcare $(22 \%)$. This highlights a strong disparity between public and private entities: when grouped together, public entities (local government, central government, healthcare, justice, and regulators) account for 46 out of the 60 entities (77\%).

Of the 60 cases, only 54 have information regarding the number of records affected by the breach. The total number of records breached in ICO civil penalty cases between 2010 to 2017 is over nine million. Table 1a shows that security failure accounts for nearly half of all total records (48\%). Disclosure error, which is the most frequent cause of data breach within the data set, is the second largest contributor providing $27 \%$ of the breached records.

The total fines awarded match the count of causes in Table 1b. For example, disclosure error fines represent $45 \%$ of all fines given, followed by loss and disposal. The most frequent cause, disclosure error, has the 

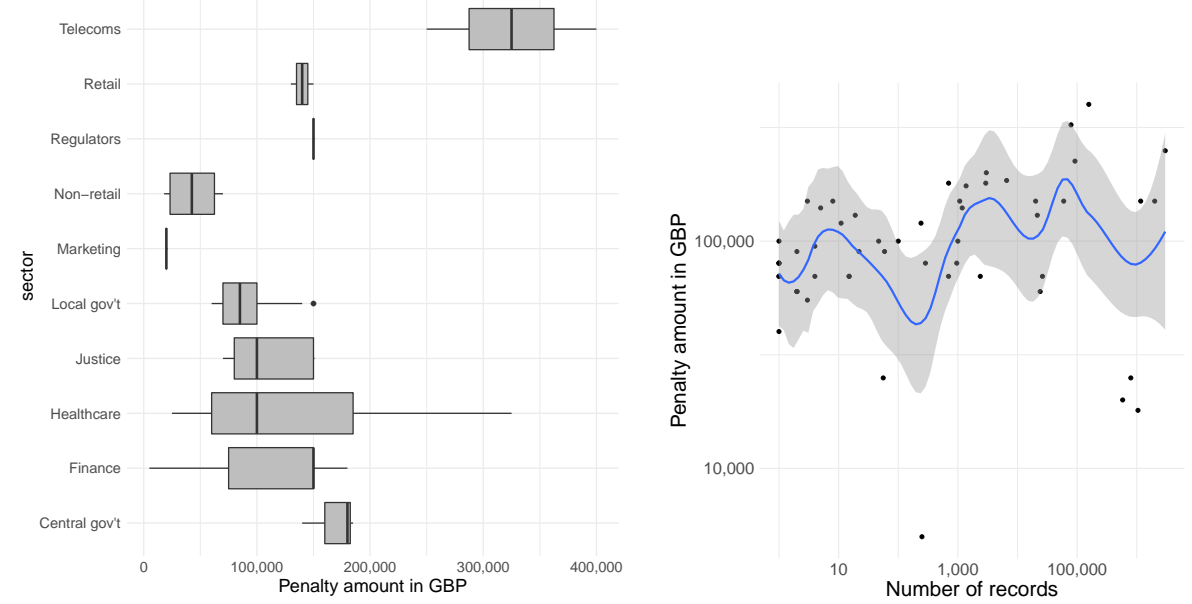

(a) Boxplot of civil penalties issued by sec- (b) Relationship between total number of tor. records and fines for data breaches $n=54$.

Fig. 1: Analysis of ICO civil penalties.

lowest average fine: $£ 92,666$. The average disclosure error fine $(£ 92,667)$ is less than half that of the security failure $(£ 266,667)$. Telecommunications and technology services have the highest mean of fines $(£ 325,000)$.

As observed in Figure 1a, the majority of fines remain under $£ 100,000$, with few fines exceeding $£ 200,000$. When considering sectors in which the fines are levied, it becomes evident that the most fined sector - local government - largely receives fines of just $£ 100,000$. When considering total fine amounts by sector, the fines levied against central government eclipses those in other sectors. The density of fines do not surpass $£ 200,000$.

Telecommunications and technology companies have received a high quantum of penalties when compared to other sectors, although these are only account for four of the fines. The largest fine (of $£ 400,000$ ) was given to TalkTalk, a telecommunications provider, for failing to take precautions against a simple SQL injection attack [9]. Healthcare has the largest range of fines, with a minimum of $£ 25,000$ and a maximum of $£ 325,000$ in Figure 1a. This sector is also associated with the most causes - which is unique as most of the sectors are associated with a single cause. The fines received by technology and telecommunications data controllers is confined to the upper limits of the range of fines.

In some popular surveys on data breaches, there is a claim that the number of records has a determination on the financial impact on the 


\begin{tabular}{|c|c|c|c|c|c|}
\hline \multicolumn{2}{|c|}{ Category Count } & \multicolumn{2}{|c|}{ Records Mean records } & \multicolumn{2}{|c|}{ Total fines Mean fine } \\
\hline sensitive & 36 & 208,242 & 5,790 & $£ 3,905,000$ & $£ 108,472$ \\
\hline general & 18 & $8,880,836$ & 493,380 & $£ 1,548,000$ & $£ 122,111$ \\
\hline Total & 54 & $9,089,078$ & NA & $£ 6,103,000$ & NA \\
\hline
\end{tabular}

(a) Category of personal data by records and fine amount.

\begin{tabular}{llll}
\hline Cause & Category & Total fines Count \\
\hline $\begin{array}{l}\text { Disclosure sensitive } \\
\text { error }\end{array}$ & $£ 2,125,000$ & 22 \\
& general & $£ 493,000$ & 7 \\
\hline Theft & sensitive & $£ 530,000$ & 6 \\
& general & $£ 305,000$ & 3 \\
\hline Loss & sensitive & $£ 300,000$ & 3 \\
& general & $£ 470,000$ & 4 \\
\hline
\end{tabular}

(b) Comparison of causes by category.

Table 2: Tables comparing data breaches, controlling for type of personal data (a) and comparison of shared causes (b). Fine amounts are in GBP.

organisation. ${ }^{7}$ We examine this claim in relation to regulatory action using local polynomial regression [4]. Figure $1 b$ shows number of records has a very marginal effect on the total quantum of the fine.

In Section 2.2, we distinguished between sensitive personal data and a broader general class. Within the civil penalties data set, there are 54 cases with available information on the number of records and types of case. Table 2a shows that more than two-thirds of fines (68\%) are associated with sensitive data and comprise more than half of the total fines (57\%). The mean fine and mean records illustrate a strong indication that context is more influential than the number of records breached in determining the final penalty amount. Sensitive records account for $2.3 \%$ of the total amount of records breached, but comprise $64 \%$ of the total fines.

When considering only those causes that are present in both sensitive and general breaches, this includes only disclosure error, theft and loss. Table $2 \mathrm{~b}$ shows that disclosure error penalties have been issued three times more than for disclosure errors of general data. Disclosure errors of sensitive data constitute $65 \%$ of penalties levied for sensitive data and $37 \%$ of all penalties levied.

\footnotetext{
${ }^{7}$ Ponemon Institute: Cost of Data Breach Study: United Kingdom. http://www-03. ibm.com/security/data-breach/
} 


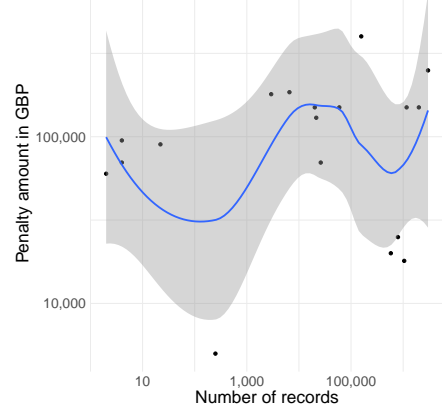

(a) General personal data, $n=18$.

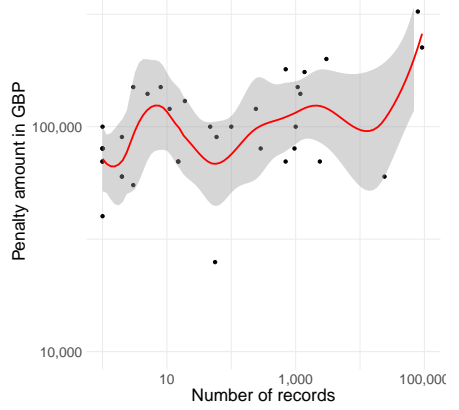

(b) Sensitive personal data, $n=36$.

Fig. 2: Relationship between amount of records breached and fines, controlling for category of data.

To further explore the number of records and fines, general and sensitive personal data were separated and their corresponding relationship between number of records and fines are analysed in Figure 2. When controlling for category of data breached, the upward trajectory is more pronounced for sensitive data (see Figure 2b). While the amount of records has somewhat of an effect on quantum of penalty, this relationship seems stronger within sensitive data breaches.

\subsection{Data breach reporting}

Using the second data set, we examine the types of cases that have been reported to the ICO in regards to data breach. Over the course of a year, the ICO received 1,895 reports of data breaches within the UK. Table 3 shows that the largest category ( $24 \%$ of all reports) are listed as some other type of data security failure. The vast majority of reports have to do with data mismanagement, with causes ranging from loss of media or paperwork to inadvertent disclosure via email, posting or conversation. Failures of security, including the consequences of hacking, account for $8 \%$ of the reported breaches. Within this data set, there is no indication of the number of records that have been affected.

Although forming a small portion of data breach reports, security failures has been a primary concern in the literature (see, for example, [13]). Within security failures, general business is a source of a quarter of all reports, with telecommunications forming $11 \%$ of reports, and retail accounting for $10 \%$. The low representation of security breaches may be due to the lack of obligation on the part of data controllers to report 


\begin{tabular}{lr}
\hline Cause of data breach & Count \\
\hline Loss or theft of paperwork & 355 \\
Post to incorrect recipient & 347 \\
Email to incorrect recipient & 248 \\
Loss or theft of device & 108 \\
Hacking & 158 \\
Insecure disposal of paperwork & 77 \\
Failure to redact & 75 \\
Information uploaded to website & 38 \\
Verbal disclosure & 27 \\
Insecure disposal of hardware & 5 \\
Other principle 7 failure & 457 \\
\hline
\end{tabular}

Table 3: Causes of data breach April 2015 to March 2016, $n=1,895$.

\begin{tabular}{lcl}
\hline Sector & Penalty amount (GBP) & Cause of data breach \\
\hline Healthcare & 185,000 & Improper disclosure of details of staff. \\
Finance & 180,000 & Theft and loss of servers containing customer data. \\
Healthcare & 25,000 & Personal details of patients were erroneously emailed. \\
Police & 80,000 & A victim's personal details were passed to the suspect in error. \\
\hline
\end{tabular}

Table 4: Description of the four entities in receipt of a civil penalty during between April 2015 and March 2016.

these instances to the ICO. When correlated with the civil penalties data, there are four entities that were fined for data breaches, totalling $£ 470,000$. Table 4 describes the penalties for data breaches which arose during the reporting period. Each of the cases has improper disclosure as a cause.

\section{Discussion and conclusions}

This work provides an exploratory analysis of a novel data set, in order to evaluate how that data may facilitate analysis of data breaches. Within the monetary penalty data set, there is a noted difference between public and private entities - with the public sector being fined more often. There may be a number of reasons for this, including intelligence-gathering practices by the ICO or effective reporting mechanisms within the public sector. Other entities, such as telecommunications services, must report, but have only been fined in two instances. Security failure is the cause of the biggest loss of records within the civil penalties data set, but that may eclipse other conclusions relevant to data security practice. Negligence can be harmful as it results in small, constant losses rather than one large event. 
Poor organisational practice leads to a greater frequency of data breach incidents than external attacks. This is not meant to diminish the risk and threat of malicious, external actors, but, instead, to draw attention to a clear challenge that data controllers are experiencing in developing the appropriate operational controls to prevent negligent, internal action. In the past year, there were 158 external 'hacking' attacks reported to the ICO. This is in contrast to 595 incidents reported of posting personal data to incorrect recipients or five devices not disposed of in a correct manner or 108 reports of lost, unencrypted devices containing personal data.

Our results demonstrate that context is an important feature in a data breach. With an upper limit of $£ 500,000$, the overall average of fines is around $£ 110,000$. This figure is not meaningful in and of itself, as it covers a range from $£ 1,000$ to $£ 400,000$ (both given for security failures). The overall average fine for general data breaches was not much higher than for data breaches concerning sensitive data, despite many more records being lost in the former. Controlling for sector and cause, the sensitivity of the data appeared to increase the quantum of the monetary penalty. This may be due to the over-representation of sensitive disclosure error types of cases, especially as public sector institutions in the UK include hospitals.

It is necessary to point out the limitations of this study. First, the civil penalty data set is focused on a specific subset of data breaches. The fines are levied where the ICO has deemed that the data controller's actions did not reach an adequate level of diligence. Therefore, only a small portion of reported breaches are fined, as demonstrated in the two analysed data sets. It should be noted that, while negligence is a factor, data breaches may occur even when such diligence is observed. Second, the wide scope of the term data breach may include factors and considerations that are not present in other studies.

The results show that privacy is more affected by gaps and failures of operational practice than from attackers. While security failures have resulted in more records being lost, the frequency of non-security causes of data breaches is much higher. This highlights that data security is not in and of itself sufficient to address the challenges within data privacy.

\section{Acknowledgements}

AC would like to thank the EPSRC and the Oxford Radcliffe Scholarship for financial support. The authors would like to thank the anonymous reviewers for their helpful and constructive feedback. 


\section{References}

1. Acquisti, A., Friedman, A., Telang, R.: Is there a cost to privacy breaches? An event study. ICIS 2006 Proceedings p. 94 (2006)

2. Campbell, K., Gordon, L.A., Loeb, M.P., Zhou, L.: The economic cost of publicly announced information security breaches: empirical evidence from the stock market. Journal of Computer Security 11(3), 431-448 (2003)

3. Cavusoglu, H., Mishra, B., Raghunathan, S.: The effect of internet security breach announcements on market value: Capital market reactions for breached firms and internet security developers. International Journal of Electronic Commerce 9(1), 70-104 (2004)

4. Cleveland, W., Grosse, E., Shyu, W.: Statistical Models in S, chap. Local regression models, pp. 309-376. Chapman \& Hall, London (1991)

5. Edwards, B., Hofmeyr, S., Forrest, S.: Hype and heavy tails: A closer look at data breaches. Journal of Cybersecurity 2(1), 3-14 (2016)

6. Heitzenrater, C.D., Simpson, A.C.: Policy, statistics and questions: Reflections on UK cyber security disclosures. Journal of Cybersecurity 2(1), 43 (2016), thttp: //dx.doi.org/10.1093/cybsec/tyw008

7. Information Commissioner's Office: Information Commissioner's guidance about the issue of monetary penalties prepared and issued under section 55C (1) of the Data Protection Act 1998. (2015), https://ico.org.uk/media/1043720/ ico-guidance-on-monetary-penalties .pdf

8. Information Commissioner's Office: ICO Disclosure Log - Response IRQ0630777. https://ico.org.uk/about-the-ico/our-information/disclosure-log/ irq0630777/ (June 2016)

9. Information Commissioner's Office: TalkTalk Telecom Group plc Monetary Penalty Notice. https://ico.org.uk/media/action-weve-taken/mpns/ 1624087/talktalk-mpn-20160324.pdf (October 2016), https://ico.org.uk/ media/action-weve-taken/mpns/1625131/mpn-talk-talk-group-plc.pdf

10. Ishiguro, M., Tanaka, H., Matsuura, K., Murase, I.: The effect of information security incidents on corporate values in the japanese stock market. In: International Workshop on the Economics of Securing the Information Infrastructure (WESII) (2006)

11. Johnson, M.E., Willey, N.: Usability failures and healthcare data hemorrhages. IEEE Security \& Privacy 9(2), 35-42 (2011)

12. Liginlal, D., Sim, I., Khansa, L.: How significant is human error as a cause of privacy breaches? An empirical study and a framework for error management. Computers \& Security 28(3), 215-228 (2009)

13. Phua, C.: Protecting organisations from personal data breaches. Computer Fraud \& Security 2009(1), 13-18 (2009)

14. Schatz, D., Bashroush, R.: The impact of repeated data breach events on organisations' market value. Information \& Computer Security 24(1), 73-92 (2016)

15. Verendel, V.: Quantified security is a weak hypothesis: A critical survey of results and assumptions. In: Proceedings of the 2009 workshop on New Security Paradigms Workshop. pp. 37-50. ACM (2009) 